\title{
eJRIEPS
}

Ejournal de la recherche sur l'intervention en éducation physique et sport

$23 \mid 2011$

Varia

\section{Influence de l'ouverture du score sur la victoire finale en football}

\section{Eric Duprat et Etienne Birmelé}

\section{(2) OpenEdition}

\section{Journals}

Édition électronique

URL : http://journals.openedition.org/ejrieps/4570

DOI : 10.4000/ejrieps.4570

ISSN : 2105-0821

Éditeur

ELLIADD

\section{Référence électronique}

Eric Duprat et Etienne Birmelé, «Influence de l'ouverture du score sur la victoire finale en football », eJRIEPS [En ligne], 23 | 2011, mis en ligne le 01 avril 2011, consulté le 21 février 2020. URL : http:// journals.openedition.org/ejrieps/4570 ; DOI : 10.4000/ejrieps.4570

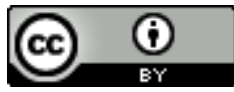

La revue eJRIEPS est mise à disposition selon les termes de la Creative Commons Attribution 4.0 International License. 


\section{Influence de l'ouverture du score sur la victoire finale en football}

\section{Eric Duprat ${ }^{*}$ et Etienne Birmelée*}

* Département STAPS de I'Université d'Evry - Val d'Essonne, chercheur associé au Gédiaps de Paris 12 - Créteil, France

** Laboratoire Statistique et Génome de l'Université d'Evry-Vald'Essonne - UMR CNRS 2071, INRA 1152, France

Résumé

Cette étude s'inscrit dans la démarche technologique présentée par Bouthier \& Durey (1994), abordée plus particulièrement dans le cadre des sports collectifs par Lemoine (2003), Mouchet (2003), Duprat (1996, 2005), Uhlrich (2005). Notre objectif est de mettre à jour les critères de performances les plus probants dans la pratique du football. Nous avons porté notre attention sur l'influence de l'ouverture du score sur l'issue de la rencontre. Notre hypothèse de travail est d'affirmer que l'équipe qui ouvre la marque a plus de chances de gagner. Nous avons donc réuni les informations dans ce domaine pour les matchs de haut niveau masculin, mais aussi féminin. Nous avons saisi les informations pour les rencontres de Coupes du Monde, de Championnats d'Europe des Nations et du Championnat de France. Les résultats obtenus confirment notre hypothèse et devraient orienter les méthodes d'intervention lors de la formation.

\section{Introduction}

Aujourd'hui, nul ne peut nier l'apport considérable des sciences d'appui dans l'évolution des pratiques sportives tant du point de vue de la recherche de performance que de celui de l'intervention lors des apprentissages initiaux, puis du perfectionnement. L'approche technologique de l'activité se présente alors comme un outil complémentaire pour aborder les pratiques sous un angle original, centré sur la réalité du terrain. Nous nous sommes plus spécialement intéressés aux paramètres d'analyse des sports collectifs et surtout du football. Notre but est de mettre en avant les critères de performances à partir desquels nous pouvons organiser l'intervention de l'entraîneur, de l'enseignant. Même si la visée de la pratique compétitive et de l'enseignement différent sur certains points, il nous semble 
que les logiques d'action qui fondent la pratique restent identiques quel que soit le secteur d'activité dès lors que l'on recherche la performance et / ou l'efficacité. L'objet de cette étude concerne l'ouverture de la marque en football. Nous voulons vérifier dans quelle mesure l'équipe qui ouvre le score a plus ou moins de chances de remporter la victoire. Nous partons du postulat que le premier objectif du jeu est la victoire. Cette idée d'aller de l'avant de chercher à marquer en premier pourrait participer à l'instauration d'une visée positive du jeu, orientée vers l'attaque. Mais se vérifie-t-elle au niveau des performances actuelles ? Marquer un but en premier est-il un gage de succès ?

\section{Cadre théorique}

Le passage incontournable du terrain au laboratoire et du laboratoire au terrain est une constante dans tous les travaux scientifiques qui se doivent d'être confrontés à la « réalité de la pratique ». Pour aider dans ce cheminement d'ingénierie, la technologie des Activités Physiques, Sportives et Artistiques (APSA) se présente comme un outil privilégié. Elle permet d'orienter son travail vers les points clefs de transformations et d'évolutions des pratiques d'intervention. Dans leur présentation de la technologie des APSA, David et al. (1999) proposent « une recherche sur le savoir technique » à partir de trois niveaux. Le premier concerne la tâche et l'activité du ou des sujets, notion déjà exploitée dans la psychologie de l'ingénierie humaine et nouvel outil dans le domaine de l'éducation. Le second s'attache plus au contexte de la tâche et aux ressources sollicitées par les acteurs au cœur même de la pratique, c'est-à-dire avec une visée « écologique » souvent reprise aujourd'hui. Le troisième s'intéresse plus au « contexte organisationnel de la tâche » et à « la signification des pratiques » déjà utilisés dans l'analyse des systèmes techniques complexes.

Lors des travaux présentés aux 4èmes Journées Alain Durey (JAD, nov. 2009), Eloi propose diverses orientations de réflexion à partir de l'article initial de Bouthier \& Durey (1994). La première concerne le registre « de lecture » et s'attache aux moyens mis en œuvre pour analyser l'activité du pratiquant, aux méthodes utilisées ainsi qu'à leur évolution. La seconde s'attache au registre « de maîtrise » et tente d'analyser les transformations vécues dans les techniques individuelles et collectives, les évolutions des techniques. Le troisième registre dit « de participation » vise à étudier les autres acteurs de la production technique et leur influence sur la pratique, telle que les conséquences de l'évolution des règles. Enfin, la quatrième orientation appelé registre « de transformation » étudie plus précisément le domaine de l'intervention et des procédures de transmission mises en œuvre, ainsi que les visées de transformations chez le pratiquant. 
Notre contribution à ces journées (JAD) consiste à présenter un travail réalisé dans le domaine de la lecture du jeu et l'analyse des faits. Nous considérons cet aspect comme essentiel dans la compréhension des déterminants de l'action et de la production technique intégrée à un ensemble collectif et coopératif. C'est à partir des éléments observés et des invariants constatés ou des probabilités de production que l'on peut confirmer ou infirmer la pertinence de l'action suivant les configurations du jeu. C'est en se basant sur ces constats que l'on peut aborder les phénomènes liés à l'évolution de la pratique et ses conséquences sur le jeu, à l'instar de l'évolution des systèmes de jeu. Cela permet une remise en cause favorable aux transformations nécessaires et une lutte productive face au dogme de certains protagonistes adeptes d'un jeu austère. Cela permet aussi de confirmer, tant que les lois du jeu restent en l'état, certains principes de jeu intangibles présentés depuis 1967 par Wade, repris par Duprat en 2007. C'est donc dans cette optique que nous inscrivons notre étude.

1. 1. Au moins un but

John Wesson (2004), en se basant sur la Premiership anglaise, aborde les probabilités d'une équipe à marquer un but lors d'un match. II démontre aussi que la stratégie défensive adoptée par les équipes considérées comme plus faible est pertinente. En effet, les statistiques montrent qu'une équipe a de grandes chances de marquer au moins un but durant le temps réglementaire. Le fait de défendre et d'empêcher l'adversaire de marquer serait un facteur de rééquilibrage des chances. "II est clair qu'un match à score faible avantage l'équipe faible et qu'un match à score élevé profite à l'équipe forte » (op.cité p. 85). Deux idées fortes ressortent malgré tout de ses travaux. La première est l'importance de toujours chercher à améliorer sa moyenne de buts marqués par unité de temps quel que soit le niveau de l'équipe adverse. La seconde concerne la nécessité de toujours être dans cette démarche d'accroître le score même si le risque augmente de prendre un but. Ces deux orientations nous semblent essentielles et apportent un premier éclairage sur les « choix philosophiques » pris par les entraîneurs. La dernière Coupe du Monde (2010) illustre bien la tendance actuelle pour un jeu tourné vers l'offensive, puisque l'Espagne (vainqueur) et l'Allemagne appréciée pour son jeu vers l'avant ont marqué cette compétition, à l'inverse de notre sélection nationale.

Par ailleurs, les chiffres présentés par Duprat (2005), pour la Coupe du Monde de 1998 en France, confirment l'idée que l'ouverture de la marque est importante puisque la moyenne des buts marqués lors d'une rencontre s'élève à 2,75. Cela implique que chaque équipe marque en moyenne 1,4 but par rencontre. Si l'on tient compte des matchs nuls à $0-0$, et 


\section{eJRIEPS 23 avril 2011}

qu'une des équipes peut marquer plusieurs buts, l'influence du premier but se trouve renforcée.

1. 2. Différences des résultats

Les informations issues des observations de Michel Ebe (1988) vont nous apporter certains indices complémentaires. Dans une première approche commune (non publiée) nous avons étudié les résultats d'un grand nombre de rencontres (cf. tableau1). Entre 2000 et 2006, nous avons relevé les résultats dans trois types de compétitions différentes : le championnat de France de Ligue 1 professionnelle, la Champion's League et les rencontres internationales opposant les équipes nationales en compétition ou en matchs «amicaux ». L'ensemble représente 1160 confrontations dont 24,3\% de matchs nuls pour $75,7 \%$ de matchs gagnés (dont 71,6 \% avec un écart de un à trois buts).

Tableau 1. Récapitulatif des résultats observés

\begin{tabular}{|l|l|l|}
\hline Scores & Nombres de matchs & Pourcentages \\
\hline Match nul & 282 & $24,3 \%$ \\
\hline Victoire avec +1 & 492 & $42,4 \%$ \\
\hline Victoire avec +2 & 252 & $25,2 \%$ \\
\hline Victoire avec +3 & 86 & $7,4 \%$ \\
\hline Victoire avec +4 à +8 & 48 & $4,1 \%$ \\
\hline
\end{tabular}

Nous pouvons déjà observer que dans 42,4 \% des cas, au minimum (score de 1-0), l'équipe qui ouvre la marque remporte la rencontre. Ce chiffre est largement supérieur au taux des matchs nuls (24,3\%). Nous constatons aussi, sans prendre en compte l'ouverture du score, qu'on remporte le match dans $36,7 \%$ des cas lorsque l'écart s'élève à deux buts ou plus. Si l'on supprime les matchs nuls cela représente prêt de $44 \%$ des matchs gagnés ce qui montre l'intérêt de jouer pour marquer.

\section{3. Dernière Coupe du Monde}

Une étude que nous venons de réaliser (non publiée) sur la dernière Coupe du Monde de football (2010) en Afrique du Sud montre que sur 64 rencontres, 8 se sont soldées par un 0-0 et 54 ont vu le tableau d'affichage évoluer. Sur l'ensemble des rencontres, l'équipe qui a ouvert la marque a gagné dans $67,2 \%$ des cas, a fait match nul dans $15,6 \%$ des cas, et a perdu dans $4,7 \%$ des cas. Si l'on tient compte seulement des matchs avec au moins un but marqué, le taux de victoires après l'ouverture du score passe à 76,8 \%, le taux de matchs nuls à $17,9 \%$ et le taux de défaites à 5,4\%. Ajoutons que lorsqu'une équipe a 


\section{eJRIEPS 23 avril 2011}

marqué deux buts, sans en concéder un, elle a gagné dans $100 \%$ des cas. Dix sept matchs se sont terminés sur le score de $1-0$ soit $26,5 \%$ de l'ensemble et six, soit 9,4\%, sur le score de 2-0. Les rencontres où une seule équipe marque un ou deux but(s) représentent donc 35,9 \%. On peut déjà considérer l'ensemble de ces chiffres actuels comme suffisamment révélateurs d'une tendance forte à la victoire pour l'équipe qui marque le premier but. A partir de ces différents résultats, nous avons poursuivi notre exploration pour essayer de vérifier notre hypothèse.

\section{Méthode}

La première étape a consisté à relever les scores des rencontres sur un nombre de matchs suffisamment important pour que les chiffres puissent nous indiquer des tendances. Nous avons recueillies les données pour cent rencontres de championnat de la Ligue 1 Française (CF-m, masculin) lors de plusieurs journées de compétition. En fait, nous avons laissé passer le début de la saison qui correspond à un ajustement collectif des équipes, puis nous avons choisi les journées de compétitions suivantes (10è, 12è, 14è, 16è, 18è, 20è, 22è, 24è, 26è, 28è, journées), lors de la saison 2008-2009. Cela représente un échantillon de 100 matchs. Dans le prolongement de la première étude abordée et dans la même idée nous avons pris les résultats des compétitions internationales. D'abord au niveau européen avec les résultats des Championnats d'Europe (CE-m) des Nations 2000 et 2008 ce qui représente un échantillon de 62 matchs. Puis en rassemblant les résultats de la Coupe du Monde (CM-m) de football de 2006, soit 64 rencontres.

Impliqué dans le football féminin de haut niveau, il nous est apparu intéressant, en deuxième étape, de reproduire la démarche dans le cadre de la pratique féminine, celle-ci étant rarement abordée. Précisons qu'il y a moins de changements dans les équipes féminines d'une année sur l'autre ce qui simplifie la coordination des joueuses, mais aussi moins de matchs car le groupe d'élite est réduit à douze formations (22 matchs). Nous avons donc recueilli les données dès la seconde journée et en condensant la saisie des résultats au milieu de la saison, soit 90 matchs du Championnat de France de Division 1 (CF-f, féminin) lors des journées suivantes (2è, 4è, 6è, 7è, 8è, 9è, 10è, 11è, 12è, 13è, 14è, 15è, 17è 19è, 21è). Puis nous avons retenus les résultats des 40 rencontres des Championnats d'Europe des Nations (CE-f) de 2005 et 2009, et enfin 64 confrontations lors des Coupes du Monde Féminines (CM-f) 2003 et 2007 (moins de matchs chez les femmes pour des compétitions de même niveau). 


\section{eJRIEPS 23 avril 2011}

Pour l'ensemble de ces rencontres nous avons relevé les scores et l'évolution des scores pour nous permettre d'analyser l'issue en fonction de l'ouverture du score, mais aussi lorsqu'une équipe parvient à un écart de deux buts. Précisons que nous avons analysé les résultats sur les 90 minutes du temps réglementaire de la rencontre, sans tenir compte des prolongations en cas de match à élimination. De plus, nous n'avons pas pris en compte la notion de match à domicile ou à l'extérieur étant donné les différences lors des phases finales des compétitions internationales. En effet, en s'appuyant sur l'avantage du match à domicile, certaines équipes adoptent des stratégies plus ou moins offensives ce qui peut influer sur le résultat final. Ceci est valable pour les rencontres au niveau national, mais entre peu en compte pour les compétitions internationales puisque une nation seulement évolue à domicile. Le poids de ce facteur n'est pas évalué dans cette étude et peut d'ailleurs s'envisager tant au niveau de l'ouverture du score que d'un retour éventuel au tableau d'affichage.

La troisième étape a consisté à réaliser une approche statistique pour confirmer ou non l'hypothèse de départ et aborder d'autres critères que nos données nous permettent d'analyser. Nous avons ainsi vérifié s'il existe des différences entre le football féminin et le football masculin et s'il existe des différences suivant les compétitions. Le dernier calcul effectué nous a permis de signaler une différence majeure entre les footballeurs et les footballeuses au niveau du nombre de rencontres sans but.

\section{Résultats}

Pour l'ensemble des matchs de football féminin observés, nous obtenons le tableau 2 qui suit. Précisons que les pourcentages de victoires, nuls et défaites sont calculés en se basant sur les matchs avec but(s). 
eJRIEPS 23 avril 2011

Tableau 2. Répartition des rencontres pour le football féminin

\begin{tabular}{|c|c|c|c|c|c|c|c|}
\hline Comp. & Matchs & $0=0$ & Vainq. & & Victoire & Nul & Défaite \\
\hline $\begin{array}{c}\text { C.M.f. } \\
2003 \text { et } 2007\end{array}$ & 64 & 1 & 63 & & 50 & 8 & 5 \\
\hline$\%$ & $100 \%$ & $1,6 \%$ & $98,4 \%$ & & $79,4 \%$ & $12,7 \%$ & $7,9 \%$ \\
\hline $\begin{array}{c}\text { C.E.f. } \\
2005 \text { et } 2009\end{array}$ & 40 & 2 & 38 & & 28 & 5 & 5 \\
\hline$\%$ & $100 \%$ & $5 \%$ & $95 \%$ & & $73,7 \%$ & $10,15 \%$ & $10,15 \%$ \\
\hline $\begin{array}{c}\text { C.F.f. } \\
2008-2009\end{array}$ & 90 & 5 & 85 & & 65 & 10 & 10 \\
\hline$\%$ & $100 \%$ & $5,6 \%$ & $94,4 \%$ & & $76,5 \%$ & $11,75 \%$ & $11,75 \%$ \\
\hline$\%$ & 194 & 8 & 186 & & 143 & 23 & 20 \\
\hline TOTAUX & $100 \%$ & $4,1 \%$ & $95,9 \%$ & & $76,9 \%$ & $12,4 \%$ & $10,7 \%$ \\
\hline
\end{tabular}

Pour l'ensemble des rencontres de football masculin, nous obtenons le tableau 3 suivant. A nouveau, les pourcentages de victoires, nuls et défaites sont calculés en se basant sur les matchs avec but(s).

Tableau 3. Répartition des rencontres pour le football masculin

\begin{tabular}{|c|c|c|c|c|c|c|c|}
\hline Comp. & Matchs & $0=0$ & Vainq. & & Victoire & Nul & Défaite \\
\hline $\begin{array}{c}\text { C.M.m. } \\
2006\end{array}$ & 64 & 8 & 5 & & 40 & 9 & 7 \\
\hline$\%$ & $100 \%$ & $12,5 \%$ & $87,5 \%$ & & $71,4 \%$ & $16,1 \%$ & $12,5 \%$ \\
\hline $\begin{array}{c}\text { C.E.m. } \\
2000 \text { et } 2008\end{array}$ & 62 & 6 & 56 & & 43 & 6 & 7 \\
\hline$\%$ & $100 \%$ & $9,7 \%$ & $90,3 \%$ & & $76,8 \%$ & $10,7 \%$ & $12,5 \%$ \\
\hline $\begin{array}{c}\text { C.F.m. } \\
2008-2009\end{array}$ & 100 & 12 & 88 & & 58 & 21 & 9 \\
\hline$\%$ & $100 \%$ & $12 \%$ & $88 \%$ & & $65,9 \%$ & $23,9 \%$ & $10,2 \%$ \\
\hline & 226 & 26 & 200 & & 141 & 36 & 23 \\
\hline TOTAUX & $100 \%$ & $11,5 \%$ & $88,5 \%$ & & $70,5 \%$ & $18 \%$ & $11,5 \%$ \\
\hline
\end{tabular}

D'après notre échantillon, la probabilité de remporter la rencontre en général est de $\mu 0=$ 0,39 pour l'ensemble des rencontres ( 0,36 pour les hommes, 0,42 pour les femmes). Nous appliquons alors le test de Student pour les rencontres avec ouverture du score, afin de vérifier si notre hypothèse est confirmée ou non, au seuil de 0,05 . Le résultat obtenu, soit la $p$-valeur $=2,210^{-16}$, confirme sans aucun doute l'hypothèse que l'équipe qui ouvre la 
marque a plus de chance de gagner la rencontre. Cette information est valable pour le football masculin $(0,31)$ mais aussi pour les féminines $(0,36)$. Cette confirmation obtenue, nous avons voulu comparer les deux échantillons sexués afin de déceler d'éventuelles différences. En se basant sur la répartition des victoires, nuls et défaites, un test de chideux a été effectué. Le résultat obtenu ( $p=0,27)$ montre qu'il n'y a pas de différence significative entre les deux sexes. Les proportions des victoires, nuls et défaites, après l'ouverture du score, en tenant compte de l'ensemble des compétitions, ne sont pas significativement différentes. Néanmoins il existe certains écarts sur lesquels nous reviendrons. Dans la continuité de notre vérification statistique, nous avons voulu voir s'il existe des différences suivant les compétitions. Dans le cadre du football féminin le test du chi-deux donne une p-valeur = à 0,92 qui nous permet de d'affirmer qu'il n'y a pas de différence significative suivant les compétitions. Pour ce qui est des hommes même si le résultat obtenu est différent ( $p$-valeur $=0,37$ ), la conclusion est la même et aucune différence significative n'apparaît. Nous pouvons toutefois signaler qu'il existe près de deux fois et demie moins de chances pour les filles de revenir au score que chez les garçons. Cela confirme que notre hypothèse de départ est encore plus forte dans le football féminin. Notre attention a aussi été attirée, à la vue des chiffres, par la différence au niveau des matchs sans but marqué. Le résultat obtenu avec p-valeur de 0,003 prouve qu'il existe une différence significative entre le football masculin et le football féminin où le nombre de matchs sans but est nettement inférieur.

\section{Discussion}

Nous ajoutons à ces données les pourcentages obtenus lorsque l'équipe qui ouvre la marque prend un avantage de deux buts. Dans ce cas l'équipe qui bénéfice de cette avance remporte la rencontre dans presque $96 \%$ pour les femmes et dans 88,3\% chez les hommes. Le retour des adversaires pour obtenir le match nul est observé dans $2 \%$ des cas dans le football féminin et $7,45 \%$ dans le football masculin. La logique est inversée pour $2 \%$ chez les femmes et $4,3 \%$ chez les hommes, où l'équipe adverse revient au score et l'emporte.

Pour compléter ces considérations statistiques, nous avons voulu explorer les résultats pour tenter de présenter des tendances. Si l'on estime que la possibilité de revenir au score est un critère d'équilibre des forces en présence, force est de constater que cette situation apparaît plus souvent dans le football masculin que féminin. On pourrait donc penser que le niveau dans le football masculin est plus homogène. Cela se confirme lorsqu'on observe les résultats pour les rencontres à gros écarts. Ainsi on relève chez les 
femmes 67 rencontres, soit 34,5\%, qui se terminent avec 3 buts d'écart alors qu'on en dénombre 23 , soit 10,2 \%, chez les hommes, toutes compétitions confondues. Les chiffres passent à 24 , soit $12,4 \%$, dans le football féminin pour 2 , soit 0,9 \%, chez les hommes, lorsque l'écart est de cinq buts. Si on accepte cette idée on peut déjà, au regard des résultats et en croisant ces données avec les pourcentages de matchs gagnés (après ouverture du score), déceler des différences suivant les compétitions. Ainsi pour le football féminin on peut estimer que le niveau du CEf est plus homogène que le celui du CFf qui devance légèrement la CMf. En ce qui concerne le football masculin on peut estimer que le CFm est plus homogène que le $\mathrm{CEm}$ et la $\mathrm{CMm}$ qui sont proches. Cette tendance est à relativiser étant donné certains effectifs faibles compris dans nos échantillons. II est plus complexe de déterminer les écarts dans la qualité du niveau de jeu. II faut alors faire appel à d'autres indices que les observations de Michel Ebe peuvent nous permettre d'aborder. Elles seront l'objet d'études futures basées sur, par exemple, le temps de possession du ballon par équipe. On peut aussi s'interroger sur le poids de la notion de match à domicile ou à l'extérieur qui joue certainement un rôle non négligeable. De multiples éléments entrent alors en jeu tant sur les éléments liés au terrain que sur ceux liés à l'environnement du match. Cette tâche est sans doute très complexe car nous sommes face à des influences multifactorielles.

\section{Conclusion}

Cette étude nous permet de penser que même si les résultats en football présentent l'option défensive prioritaire comme une orientation efficace ponctuellement (Wesson), elle ne peut être performante dans le temps et prendre le statut d'évidence. Les résultats sur plusieurs compétitions montrent que l'ouverture de la marque s'inscrit d'ores et déjà comme un indice fiable de la performance. C'est un facteur déterminant de la victoire. Marquer un but en premier devient donc un critère, en termes de référence et de principe, à prendre en compte dans le cadre de l'intervention. Les résultats obtenus tant pour les filles que les garçons confirment nettement notre hypothèse de départ. Nous pouvons donc affirmer que de ce point de vue les pratiques féminine et masculine du football se rejoignent. Cette orientation se renforce dès lors qu'une équipe prend deux buts d'avance sur son adversaire après avoir ouvert la marque. La philosophie prônée dans le jeu doit donc correspondre à la logique interne de l'activité qui fait de la victoire le but ultime du jeu. Cette victoire étant directement liée au nombre de buts marqués, cet objectif devient premier. Les équipes qui ont marqué ou marquent encore les mémoires sont toujours celles qui ont privilégié le jeu d'attaque. Le Brésil provoque toujours plus d'engouement 
eJRIEPS 23 avril 2011

que l'Italie même si la seconde présente un palmarès enviable. La formation du joueur, l'enseignement du football et les orientations tactiques et stratégiques en compétition devraient s'orienter prioritairement vers l'attaque. De plus, il nous semble que la notion de plaisir que ce soit dans le jeu ou dans le spectacle, qui découle de la qualité offensive et du nombre de buts marqués, se pose comme une garantie de la pérennité de la pratique.

\section{Bibliographie}

Bouthier, D. \& Durey, A. (1994). Technologie des Activités Physiques et Sportives. Impulsions, 1, 95-123.

David, B., Bouthier, D., Marsenach, J., \& Durey, A. (1999). French research into the didactique and technology of physical activity and sport : an expanding new field. Instructionnal science, 17, 148-162.

Duprat, E. (1996). Technique et prise de décision : l'exemple du tacle en football. Diplôme d'Étude Approfondie, Université Paris XI, Orsay.

Duprat, E. (2005). Approche technologique de la récupération du ballon lors de la phase défensive en football, contribution à l'élaboration de contenus de formation innovants. Thèse de Doctorat, École Normale Supérieure de Cachan.

Duprat, E. (2007). Enseigner le football en milieu scolaire (collèges, lycées) et au club. Les cahiers Actio. Joinville le Pont : Actio.

Ebe, M. (1988). Le football au scanner. Edité à compte d'auteur.

Éloi, S. (2009). Présentation des registres de lectures en technologie des APSA. Communication présentée aux 4èmes Journées Alain Durey, Gédiaps de Créteil, Université Paris Est Créteil.

Lemoine, A. (2003). Étude de la production d'informations dans le cadre de la transmission instantanée du ballon en football. Thèse de Doctorat non publiée, Université de Picardie, Amiens.

Mouchet A. (2003). La subjectivité dans les prises de décisions tactiques des joueurs d'élite 1 en rugby: caractérisation et perspectives pour l'intervention. Thèse de Doctorat du $3^{\text {ème }}$ cycle, Université Paris XII Créteil.

Uhlrich, G. (2005). Rôle des artefacts matériels et cognitifs dans la formation à l'appréciation des futurs intervenants éducatifs en rugby. Thèse de l'Université Victor Ségalen, Bordeaux 2.

Wade, A. (1967). The football association : guide of training and coaching. London, EP Publishing LTD, Heinemann.

Wesson, J. (2004). La science du football. Collection Pour la science. Paris : Belin. 